Mirella Mazzeo

\title{
SCHIAVITÙ E TRATTA DI ESSERI UMANI: NUOVE CATEGORIE PER ANTICHE PIAGHE
}

\section{Premessa}

Avete mai visto uno schiavo? La domanda è di certo provocatoria, ma non così banale come potrebbe sembrare. Purtroppo ho il fondato timore di aver visto degli schiavi, in Italia, nel 2010, e di aver provato a difenderli nella mia attività lavorativa di avvocato, rendendomi ben presto conto delle gravi lacune dell'attuale corpus normativo italiano nella loro tutela. Mi rendo conto che le mie affermazioni possono apparire incredibili ed eccessive, ma la cronaca è riuscita a superare ogni immaginazione. Mi riferisco agli stranieri di Rosarno, in particolare agli sviluppi giuridici delle note vicende.

Per quanti non vivono in Italia riassumerò brevemente le vicende di cronaca. A Rosarno ${ }^{1}$ si trovavano circa cinquemila stranieri, immigrati irregolari, che lavoravano alla raccolta degli agrumi per il tramite di intermediari, forse legati alla criminalità organizzata. I clandestini lavoravano per dodici ore al giorno, senza cibo né acqua, per ottenere la cifra di circa 20 euro al giorno ${ }^{2}$. Vivevano in capannoni sprovvisti di acqua, luce, gas, fognatura: semplici ammassi di lamiera privi di suppellettili e riscaldamento. A gennaio alcuni immigrati si sono ribellati chiedendo che la retribuzione fosse a cottimo non solo nel caso di mancato raggiungimento di standards di efficienza minimi, ma anche in caso di particolari abilità tecniche, ossia il rispetto dei patti stretti coi "caporali". La reazione alle loro richieste è stata una sparatoria contro gli stranieri. Gli immigrati, quindi, si sono uniti, hanno dato luogo ad una rivolta e, armatisi di spranghe e bastoni, hanno per giorni recato disordini nella cittadina e scontri con gli italiani. Per riuscire a riportare la legalità vi è stato un for- 
te intervento dello Stato che, tramite le Forze dell'Ordine, ha prelevato gli stranieri al fine di proteggerli ${ }^{3}$.

Il problema è che tali stranieri erano tutti irregolari, pertanto soggetti alla pena per il reato di clandestinità e destinati ad essere ospitati presso un Centro di Identificazione ed Espulsione (CIE) fino al rimpatrio forzato nei Paesi d'origine, poverissimi Stati dell'Africa Subsahariana. Il $\mathrm{CIE}^{4}$ non è un carcere, ma è il luogo dove si attua la detenzione amministrativa. Gli ospiti del CIE non possono uscire dalla struttura, da un punto di vista architettonico del tutto analoga ad un carcere, dove l'ordine viene mantenuto grazie a personale militare armato5. Per evitare che i continui episodi di autolesionismo all'interno della struttura portino a conseguenze irreparabili il CIE è sprovvisto di rubinetti, chiodi, bulloni, viti, lamette, bastoni e ogni altro oggetto utilizzabile per ferirsi. Tuttavia, come si entra, è incessante il rumore di quanti si scagliano contro le porte antisfondamento e le pareti. La disperazione viene tenuta sotto controllo con l'ausilio di personale specializzato in psichiatria. Gli avvocati durante i colloqui con gli ospiti sono controllati da almeno due militari armati a distanza molto ravvicinata. All'interno della struttura non sono ammessi visitatori di sorta, in particolare i giornalisti. Possono visitare i CIE esclusivamente i parlamentari, previa autorizzazione prefettizia.

A fronte della terribile situazione dei migranti a Rosarno credo che il dubbio sull'attuale esistenza della schiavitù sia lecito.

Il mio intervento, che si dipanerà in un continuo confronto tra antichità, modernità ed attualità pratica, spero riuscirà a fornire degli spunti di riflessione, nella consapevolezza della mancanza di risposte definitive.

\section{La dominica potestas sui servi e la nuova schiavitù}

La schiavitù, intesa come diritto di proprietà di un essere umano su un altro essere umano, è stata da lungo tempo abolita in ogni angolo del globo terrestre ${ }^{6}$. Inol-

3 Cfr. per una sintesi degli avvenimenti "Schiavi a Rosarno per due euro l'ora", Giovanni Bianconi, in "II Corriere della Sera", 27 aprile 2010 e "Rosarno: 30 arresti per caporalato" , Marco Ludovico, in "Il sole 24 ore", 27 aprile 2010. Sul ruolo della criminalità organizzata nella vicenda vedasi per tutti "Immigrati in mano alle "ndrine", Francesco Condoluti, in "ll sole 24 ore", 31 marzo 2010. Di opposta opinione "Altro che 'ndrangheta", Giacomo Amadori in "Panorama", 6 maggio 2010.

4 I CIE sono previsti dall'art. 14 D. Lgs 286/98 (T.U. Immigrazione), come modificato dall'art. 12 L. 189/2002. \|I D. L. n. 92/2008 disciplina tali strutture.

5 E' vietato fotografare e/o riprendere l'interno del CIE. Nelle zone destinate alla permanenza degli ospiti non possono accedere neanche i legali. Immagini e video di denuncia delle condizioni di vita all'interno di tale struttura sono visionabili in <<radioradicale.it/riprese-esclusive-da dentro-il cie-di bari-immagini-di uno stato-illegale>>. II 5 maggio 2010 è iniziata una class action procedimentale nei confronti delle pubbliche amministrazioni e un'azione partecipativa dei cittadini a tutela dei diritti fondamentali dell'uomo, finalizzata al riconoscimento dei diritti civili per i migranti ristretti nel $\mathrm{CIE}$ di Bari. Cfr. <<classactionprocedimentale.it>> 
tre, non bisogna confondere la schiavitù con forme più o meno brutali di lavoro forzato, di sfruttamento, di debolezza socio-economica e di prostrazione psicologica.

Lo studio della schiavitù in epoca antica esula dagli obiettivi di questo intervento, necessariamente contenuto, ma è opportuno richiamare in estrema sintesi alcuni concetti fondamentali.

Nel diritto romano lo schiavo era una res: il servus non era soggetto di diritto, ma solo oggetto.

Il solo parlare di "schiavitù in periodo romano" è viziato da una prospettiva distorta: infatti mentre la modernità parte dal punto di vista dell'essere umano tenuto in stato di schiavitù, il diritto romano disciplinava la dominica potestas sui servi, ossia si preoccupava di regolamentare il diritto del proprietario dello schiavo, secondo una prospettiva invertita rispetto alla nostra.

Tale concezione emerge nelle affermazioni di Gaio:

"summa divisio personarum hac est, quod omnes homines aut liberi sunt aut servi"?.

Successivamente il giurista si occupa degli status delle persone, incluso quello dei servi ${ }^{8}$, che erano una res mancipi ${ }^{9}$. L'insigne giurista si preoccupa subito di sottolineare che non era lecito "infierire in maniera eccessiva e senza motivo sui propri schiavi" colui che uccide senza motivo il proprio schiavo è responsabile alla stregua di colui che uccide uno schiavo altrui"' ${ }^{2}$. Tuttavia, ciò accade non già per un ipotetico autonomo diritto dello schiavo, ma perché "non è lecito fare cattivo uso di un nostro diritto" 13 , come avviene per i prodighi, cui viene interdetta l'amministrazione dei beni ${ }^{14}$.

La condicio servilis si protraeva a tempo indeterminato e si tramandava nelle famiglie servili, ma era trasformabile attraverso la manumissio. La forza originaria della condizione di schiavo si riflette anche in caso di manomissione, per il particolare rapporto che il liberto aveva con il suo patronus, che, tra l'altro, non poteva accusare $^{15}$.

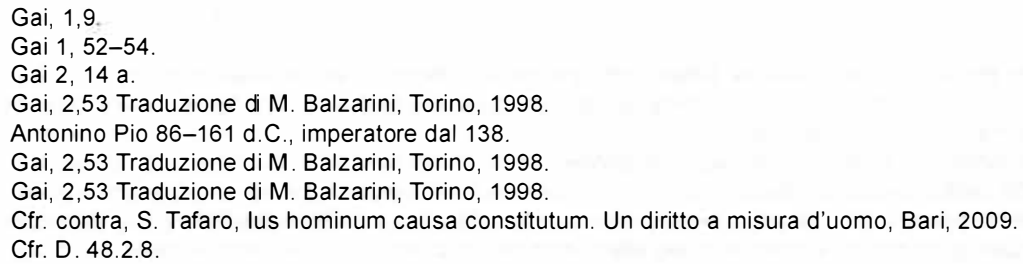


Conseguenza della condizione di res dei servi erano la preclusione della possibilità di contrarre matrimonio ${ }^{16}, l^{1}$ 'assenza di potestà sui figli, dei quali il diritto si interrogava se fossero fructus ${ }^{17}$.

Ovviamente, come le altre personae in protestate, lo schiavo di regola ${ }^{18}$ non poteva essere titolare di rapporti giuridici, e su di esso il dominus aveva il ius vitae ac necis, il ius vendendi e il ius noxae dandi.

La condizione servile da un punto di vista giuridico apparentemente uniforme era di fatto estremamente variegata da un punto di vista sociale: tra gli schiavi della familia rustica, impiegati nei lavori agricoli più duri e gli schiavi intellettuali nelle grandi famiglie romane le differenze erano abissali. Ovviamente anche l'evoluzione storica ebbe un peso notevole e il cristianesimo influì sulle limitazioni ai poteri del dominus e sulla semplificazione delle manomissioni ${ }^{19}$.

Ciò che vorrei sottolineare è la concezione giuridica antica in base alla quale un essere umano poteva essere in proprietà di un altro e del tutto spogliato di autonomi diritti. Il problema del diritto romano era disciplinare il diritto del dominus sul servus, le manomissioni, la condizione dei liberti, ma non quella dei servi.

Una tale concezione è lontanissima da quella cristiana espressa in Col. 3,11 "Greci e Giudei, Sciti e barbari, liberi e schiavi, uomo o donna, costituiscono infatti una sola cosa in Gesù Cristo". Sarà l'evoluzione di tali concetti ed idee a condurre, attraverso un lunghissimo percorso, magistralmente enucleato dal $\mathrm{Bucci}^{20}$, all'egualitarismo di stampo moderno, con il riconoscimento dei diritti inviolabili dell'uomo, come limpidamente espresso dall'art. 2 Cost.:

"La Repubblica riconosce e garantisce i diritti inviolabili dell'uomo, sia come singolo sia nelle formazioni sociali ove si svolge la sua personalità...".

\section{II diritto penale e la nuova schiavitù}

Sembra impossibile che oggi possano esserci persone in condizioni analoghe a quelle che caratterizzavano l'antica schiavitù. Eppure il diritto non ignora che ciò possa avvenire. La condanna del fenomeno schiavile a livello mondiale è nota e si è sviluppata per tappe. Fin dal 1462 il Papa Pio II definì la schiavitù un magnum scelus, ma solo nel 1807 la Gran Bretagna, maggiore potenza marina dell'epoca, abolì 
il commercio degli schiavi, e ancora nel 1815, durante il Congresso di Vienna, il Papa Pio VII chiese la proibizione del commercio di schiavi.

Numerose convenzioni internazionali ${ }^{21}$ proibiscono la schiavitù, stigmatizzata in modo esemplare dall'art. 4 della Dichiarazione Universale dei Diritti dell'Uomo del 1948:

"Nessun individuo potrà essere tenuto in stato di schiavitù e servitù; la schiavitù e la tratta degli schiavi saranno proibite sotto qualsiasi forma".

Per ottemperare a tali disposizioni soprannazionali e per evitare che potessero ripetersi le tristi situazioni dell'antichità la schiavitù è penalmente sanzionata. L'art. 600 c.p. punisce "Chiunque esercita su una persona poteri corrispondenti a quelli del diritto di proprietà ovvero chiunque riduce o mantiene una persona in uno stato di soggezione continuativa, costringendola a prestazioni lavorative o sessuali ovvero all'accattonaggio o comunque a prestazioni che ne comportino lo sfruttamento" e spiega che "la riduzione o il mantenimento nello stato di soggezione ha luogo quando la condotta è attuata mediante violenza, minaccia, inganno, abuso di autorità o approfittamento di una situazione di inferiorità fisica o psichica o di una situazione di necessità,o mediante la promessa o la dazione di somme di denaro o di altri vantaggi a chi ha autorità sulla persona". Nonostante la formula ampia della fattispecie, di regola viene riconosciuta unicamente in casi di sfruttamento della prostituzione caratterizzati da una forte violenza psichica e fisica sulle donne, in quanto condizione analoga alla schiavitù. E' importante sottolineare che il consenso dell'offeso non giustifica il fatto, essendo lo status libertatis un diritto di cui il titolare non può disporre fino al punto da permetterne la completa soppressione ${ }^{22}$.

Sono altresì punite la tratta di persone (art. 601 c.p.) e l'acquisto e alienazione di schiavi (art. 602 c.p.).

Ci sembrerebbe di trovarci di fronte ad un quadro rassicurante, ma purtroppo la schiavitù continua ad esistere, sotto altre forme.

Kevin Bales, massimo esperto mondiale di schiavitù contemporanea, insegna che "Si può pensare che la schiavitù sia una questione di proprietà, ma dipende da ciò che intendiamo per proprietà. In passato il rapporto di schiavitù presupponeva che una persona ne possedesse legalmente un'altra, ma la schiavitù moderna è diversa. Oggi la schiavitù è illegale ovunque e non esiste più alcuna forma legale di proprietà di un essere umano. Oggi coloro che comprano degli schiavi non chiedono una ricevuta o un certificato di proprietà, eppure ne ottengono il control-

Tra queste: Convenzione di Ginevra del 1929, Dichiarazione Universale dei Diritti dell'Uomo del 1948, Convenzione europea per la salvaguardia dei diritti dell'uomo e delle libertà fondamentali del 1950, Patto internazionale sui diritti civili e politici del 1966.

Cfr. Conf. Assise Roma 14 luglio 1968, in Giust. Pen. 1969, vol. II, P. 865. 
lo - e ricorrono alla coercizione per mantenerlo. I detentori di schiavi hanno tutti i benefici della proprietà senza averne i fastidi legali. In effetti, per i detentori di schiavi, non averne la proprietà legale si rivela un vantaggio perché esercitano su di loro il controllo totale senza avere alcuna responsabilità per ciò che possiedono" ${ }^{923}$. L'insigne autore rileva che mentre la vecchia schiavitù, sul modello di quella romana aveva determinate caratteristiche ${ }^{24}$, la nuova schiavitù ne ha altre, non meno insidiose: l'evitare la proprietà legale, il bassissimo costo d'acquisto dello schiavo e i suoi elevatissimi profitti ${ }^{25}$, il surplus di potenziali schiavi, il rapporto di breve periodo che porta agli schiavi "usa e getta".

Tuttavia, si potrebbe obiettare che in uno Stato di diritto come l'Italia una persona tenuta in tali condizioni di schiavitù potrebbe comunque rivolgersi alle forze dell'ordine, denunciare gli sfruttatori ed esser protetta e tutelata. Tutto ciò avviene per le prostitute, che hanno diritto di accedere a specifici programmi di protezione ${ }^{26}$. Ma cosa avviene per gli immigrati clandestini tenuti in stato di schiavitù?

\section{Un esempio di schiavitù in Italia: gli stranieri a Rosarno}

Gli stranieri presenti ai valichi di frontiera senza i requisiti richiesti dalla normativa sull'immigrazione sono respinti dalla Polizia di Frontiera in forza dell'art. 10 D.Lgs. 286/1998. Gli stranieri che entrino o soggiornino sul territorio nazionale senza essere in regola con la normativa vigente in materia di immigrazione integrano il reato di clandestinità e sono soggetti ad espulsione amministrativa ${ }^{27}$. L'espulsione amministrativa per lungo tempo si è rivelata un mezzo inidoneo a fronteggiare il fenomeno dell'immigrazione clandestina, poiché i migranti colpiti da tale provvedimento non lasciavano spontaneamente il territorio nazionale: sono stati pertanto istituiti i Centri di Identificazione ed Espulsione. In tali strutture gli stranieri irregolari sono trattenuti su disposizione del Questore, convalidata dal Giudice ${ }^{28}$ nel contraddittorio con le parti. Il trattenimento è disposto "per il tempo strettamente necessario" 29 e, sommando tutte le proroghe non può comunque superare la durata di 180 giorni, ossia circa sei mesi.

Cfr. K. Bales, I nuovi schiavi. La merce umana nell'economia globale. Trad. It. Maria Nadotti, Milano, 2008. Proprietà legale accertata, alto costo d'acquisto, bassi profitti, scarsità di potenziali schiavi, rapporto di lungo periodo, schiavi mantenuti a vita, importanza delle differenze etniche e di cittadinanza.

Si calcola ad esempio che l'utile procurato da una prostituta schiava tailandese superi l' $850 \%$ annuo. Cfr. K. Bales, op. cit., pp. 55-62.

Il Dipartimento per le Pari Opportunità - Presidenza del Consiglio dei Ministri finanzia, unitamente alle Regioni tali programmi di protezione e reinserimento. Cfr. art. 13 D.Lgs $286 / 1998$

La competenza in materia di immigrazione, trattenimenti e proroghe del trattenimento è del Giudice di Pace, una magistratura non togata.

Art. 14 D.Lgs 286/1998. 
Secondo alcuni i CIE "costituiscono di fatto strutture carcerarie extra ordinem" ${ }^{30}$, di certo comportano una limitazione della libertà personale che impone il controllo giurisdizionale sull'ingresso e la proroga del trattenimento presso tali strutture.

Vi è di più: l'immigrato clandestino non può prendere in locazione un'abitazione perché ciò è proibito dall'art. 12 c. 5 bis del D.Lgs $286 / 1998^{31}$, non può stare illegalmente sul territorio dello Stato, pena il reato di clandestinità, non può stipulare alcun contratto poiché la controparte, italiana o straniera che sia, altrimenti rischia un'accusa per favoreggiamento dell'immigrazione clandestina. Per chiarire: un datore di lavoro che voglia stipulare un contratto di lavoro regolare con uno straniero clandestino che già si trovi in Italia non può farlo ${ }^{32}$, in quanto il cd. decreto flussi, che regola l'ingresso regolare dei migranti in Italia presuppone che lo straniero si trovi nel suo Paese d'origine al momento dell'assunzione (art. 3 c. 4 D. Lgs 286/1998), né può stipulare un contratto di lavoro "in nero" altrimenti rischia di incorrere nel reato di favoreggiamento della permanenza clandestina. E' evidente che con il reato di clandestinità è preclusa all'immigrato irregolare la possibilità di agire in giudizio per vedere riconosciuti i propri diritti, come quella di rivolgersi alle Forze dell'Ordine, che altrimenti sarebbero costrette a restringere i migranti in un CIE.

In base all'art. 1 c. 15 L. 94/2009 gli stranieri clandestini non possono contrarre matrimonio e, comunque, lo straniero che sposi un cittadino acquista la cittadinanza solo dopo due anni dal matrimonio, sempre che il matrimonio sia ancora valido e i coniugi non siano separati. Il cosiddetto reato di clandestinità, molto controverso ${ }^{33}$, è stato introdotto dalla novella della L. n. 94/2009, cd. pacchetto sicurezza all'art. 10 bis D. Lgs n. 286/1998 (T.U. immigrazione): punisce con la pena dell'ammenda, senza possibilità di effettuare oblazione, "salvo che il fatto costituisca più grave reato, lo straniero che fa ingresso ovvero si trattiene nel territorio dello Stato, in violazione delle disposizioni del presente Testo Unico". Stessa pena è prevista per chi viola la disciplina dei soggiorni di breve durata degli stranieri per visite, affari, turismo e studio, di cui all'art. 1 L. n. 68/2007.

In sintesi: intorno agli stranieri clandestini si è fatta "terra bruciata", negando loro tutti i diritti civili ad eccezione di quello alla salute. Inizialmente vi era anche la

30 Cfr. $\ll<$ classactioneprocedimentali.it $>>$.

31 |l D.L. n. 92/2008 comportava anche la confisca dell'immobile locato, attualmente per la punibilità del locatore è richiesto l'ingiusto profitto, in base all'art. 1 c. 14 L. 94/2009 cd. pacchetto sicurezza 2009.

32 Salvo espressi provvedimenti legislativi, come la cd. sanatoria per le badanti, L. n. 102/2009.

33 Vi è stata una forte polemica sulla legittimità costituzionale di tale norma penale, che sembra sanzionare non una condotta o una omissione, ma uno status. E' opportuno sottolineare che il Capo dello Stato ha inviato al Governo una lettera in occasione della promulgazione del cd. pacchetto sicurezza, ove si esprimevano perplessità sulla tecnica legislativa adottata. La Corte Costituzionale con sentenza 5-8 luglio 2010 n. 250 ha stabilito la legittimità costituzionale della norma in esame in quanto "oggetto dell'incriminazione non è un modo d'essere della persona, ma uno specifico comportamento, trasgressivo di norme vigenti". La Consulta è stata di diverso avviso in relazione all'aggravante di clandestinità, ritenuta incostituzionale con sentenza 5-8 luglio 2010 n. 249. 
proposta di legge di obbligare i medici a denunciare i clandestini che si presentassero per essere curati, ma tale provvedimento non è stato approvato per la fiera opposizione dell'Ordine dei Medici e per fatti di cronaca che hanno dimostrato che negare il diritto alla salute a fasce di persone significa mettere in pericolo la sanità pubblica, soprattutto per quanto concerne le malattie infettive ${ }^{34}$.

\section{Conclusione}

Certamente la schiavitù intesa come proprietà legale di un essere umano sull'altro è ormai scomparsa da tempo in ogni parte del mondo, da ultimo nel 1981 in Mauritania. Tuttavia, come autorevolmente insegnato da Kevin Bales, vi sono nuove forme di schiavitù che si sostanziano non nella proprietà ma nella detenzione di esseri umani, che divengono così "merce umana usa e getta".

Sebbene la legislazione italiana punisca molto severamente la schiavitù e la tratta di esseri umani le recenti modifiche legislative hanno creato delle situazioni di soggettività giuridica ridotta, quella degli immigrati irregolari.

Come in antico avveniva per i servi oggi i clandestini non possono sposarsi, non possono stipulare contratti, non possono agire in giudizio ${ }^{35}$, non possono essere tutelati dalle Forze dell'Ordine, non vedono tutelata la loro libertà personale in quanto devono essere ristretti presso il CIE fino al rimpatrio forzato. Unico diritto riconosciuto agli stranieri è quello alla salute, ma non si comprende se ciò sia per tutelare i migranti in quanto persone, o piuttosto per tutelare la sanità pubblica ed evitare epidemie. E' vero che teoricamente i clandestini potrebbero chiedere la protezione internazionale e accedere allo status di rifugiati, ma di fatto ciò comporta un rischio elevatissimo di essere nelle more trattenuti presso il CIE ed espulsi in caso di diniego dell' istanza, ipotesi molto frequente ${ }^{36}$.

Poiché da un punto di vista giuridico i clandestini sono persone cui sono riconosciuti pochissimi diritti, finiscono con l'esser facile preda dei nuovi schiavisti, come è avvenuto a Rosarno. A Rosarno gli stranieri lavoravano in condizioni disumane, senza possibilità di stipulare validi contratti di lavoro, senza poter abitare in case di-

Emblematico a tal proposito è stato il caso di una giovane prostituta nigeriana che è morta di tubercolosi nell'autunno 2009. La ragazza, nel timore di essere denunciata e ristretta nel CIE non si è curata e, oltre ad aver così causato la propria morte, ha infettato i suoi "clienti" e le altre persone con le quali è entrata in contatto.

Se non avverso il decreto di espulsione e, in linea del tutto teorica, dati i costi dell'azione, avverso il diniego di protezione internazionale. Peraltro, salvo il caso di Patrocinio a Spese dello Stato espressamente previsto per il trattenimento e l'espulsione, non possono essere assistiti di fiducia da un legale. Infatti, non è possibile ai clandestini accedere la Patrocinio a Spese dello Stato in quanto privi di codice fiscale e per il medesimo motivo non possono retribuire il difensore, che non potrebbe rilasciare loro regolare fattura. 
gnitose, e con un tentativo di affermazione del ius vitae ac necis da parte dei "caporali" quando vi è stata la richiesta di rispettare gli accordi di lavoro. Certo, su questi migranti nessuno ha la formale proprietà, ma il fatto che i loro spostamenti fossero eterodiretti ${ }^{37}$, che la scelta di sottostare a determinati accordi lavorativi fosse priva di alternative giuridicamente lecite, e che la richiesta di aiuto si sia tramutata in detenzione amministrativa presso il CIE non è per nulla rassicurante.

I ricorsi avverso l'espulsione presentati dai difensori stanno formando una giurisprudenza di merito ${ }^{38}$, incoraggiata dalla Corte di Cassazione ${ }^{39}$, che tende a concedere la protezione ex art. 18 T.U. Immigrazione, con conseguente annullamento dell' espulsione "essendo state accertate situazioni di violenza e di grave sfruttamento nei confronti dello straniero". Tuttavia, spessissimo le decisioni dei giudici giungono quando ormai gli stranieri, trascorso il periodo massimo di trattenimento nei CIE, vengono rilasciati con consegna del foglio di via. Ciò comporta che in attesa di giudizio, che non sospende l'espulsione, i migranti dovrebbero lasciare il territorio italiano. Inoltre, rimane aperta la questione del reato di clandestinità, cui, strictu iuris, dovrebbero incorrere gli stranieri in attesa di giudizio. La durata del giudizio in casi così delicati da un punto di vista giuridico ed umano non è affatto neutra, ma può addirittura comportare la mancanza di effettività delle norme a presidio degli stranieri sfruttati. Una giustizia tardiva a volte si rivela, infatti, un diniego di giustizia, come purtroppo è accaduto per le persone che ho difeso ${ }^{40}$.

Inoltre la grande confusione che regna nell'applicazione della normativa conduce talvolta a situazioni paradossali: i giudici di pace annullano le espulsioni riconoscendo la protezione ex art. 18 T.U. Immigrazione, ma poi le questure non concedono il corrispondente permesso di soggiorno a causa di divergenze interpretative della normativa ${ }^{41}$. In questo caso gli stranieri si trovano in un vero e proprio "limbo del diritto": non possono essere espulsi, ma nemmeno vivere legittimamente in Italia perché privi di permesso di soggiorno. Pertanto a costoro è preclusa la possibilità di lavorare lecitamente, nel rispetto della legge. Non vivendo più all'interno dei CIE per scadenza dei termini di trattenimento, né essendoci strutture assistenziali specifiche, mi chiedo come possano sostenersi queste persone, visto che non possono lavorare lecitamente.

Le indagini stanno accertando lo spostamento stagionale di circa 5000 persone lungo l'asse Napoli - Reggio Calabria - Foggia. Tali spostamenti avvenivano in base a precise indicazioni dei "datori di lavoro" degli stranieri. Cfr. Giudice di Pace di Bari del 24.5.2010 cron. N. 692/10, N. 874/10 R.G. C. Cass. sez. I Ord. n. 10636 del 3.5.2010.

Nei casi concreti che ho affrontato le sentenze di annullamento dell'espulsione per concessione di protezione ex art. 18 T.U. immigrati sono giunte a distanza di vari mesi dalla proposizione delle domande, quando gli stranieri erano da tempo stati rilasciati dal CIE per scadenza dei sei mesi di termine massimo di trattenimento. Ciò signifjca che per sei mesi sono state trattenute in una struttura simile ad un carcere persone che non avrebbero dovuto esserlo. Cfr. Giudice di Pace di Bari sent. 1586/2010 del 5.10.2010 e n. 971/2010 del 27.6.2010.

41 La Questura di Bari non rilascia il permesso di soggiorno ex art. 18 perché le indagini sul presunto sfruttamento lavorativo degli stranieri sono ancora in corso; la Questura di Napoli richiede che il Giudice di Pace nella sentenza di annullamento dell'espulsione espressamente autorizzi al rilascio di permesso di soggiorno. 
Sarebbe a mio avviso opportuna una interpretazione autentica della normativa, per evitare tali situazioni paradossali

Di certo chi ha anche un solo diritto riconosciuto non può essere paragonato al servus che era esclusivamente oggetto di diritto, ma il fatto che esistano ancora oggi, in Italia, esseri umani con soggettività giuridica estremamente ridotta è inquietante. Gli effetti della applicazione concreta della normativa sugli stranieri, a causa della eccessiva durata dei processi, a volte tradiscono le aspettative di giustizia e causano inaccettabili disparità dovute alla tempistica.

A mio avviso precludere a delle persone ogni possibilità di vivere lecitamente, perché il loro stesso status di clandestini è reato, significa condurle nelle mani dei moderni schiavisti. Inoltre, ritengo che uno stato di diritto, quale l'Italia, non dovrebbe permettere che la giustizia arrivi quando ormai è troppo tardi.

\section{Postilla}

La Suprema Corte di Cassazione con la sentenza n. 13767/2010 ha statuito la obbligatorietà del contraddittorio per la proroga del trattenimento dello straniero irregolare presso il Centro di identificazione ed espulsione. Da tale decisione sono scaturite nel febbraio 201142 le ordinanze della Suprema Corte di manifesta ammissibilità dei ricorsi per Cassazione avverso i decreti di proroga del trattenimento di alcuni stranieri sfruttati a Rosarno, trasferiti presso il CIE di Bari. Si rileva che tali decisioni sono pervenute quando ormai erano scaduti i termini massimi per il trattenimento dei migranti, i quali però, ancora a fine 2011, non hanno ottenuto il permesso di soggiorno. 


\section{SCHIAVITÙ E TRATTA DI ESSERI UMANI: NUOVE CATEGORIE PER ANTICHE PIAGHE}

Nell'intervento al convegno del giugno 2010 si è trattato del tema della schiavitù, interrogandosi criticamente sulla situazione giuridica dei migranti clandestini in Italia, sulla scorta di esperienze professionali legate a vicende di cronaca.

La schiavitù era, in diritto romano, uno status personale disciplinato in base ad un'ottica opposta rispetto a quella attuale: fulcro della disciplina era la dominica potestas sui servi, ossia il diritto del proprietario su quella particolare res, che era lo schiavo.

A partire dalla concezione cristiana dell'uguaglianza umana di fronte a Dio e attraverso un lunghissimo percorso che giungerà fino alla Dichiarazione Universale dei Diritti dell'Uomo del 1948, si arriverà a vietare la schiavitù e ad affermare che ogni essere umano è soggetto di diritto. Tuttavia, come sostenuto da autorevoli autori, vi sono attualmente nuove forme di schiavitù che si sostanziano non nella proprietà ma nella detenzione di esseri umani, che divengono così "merce umana usa e getta".

Sebbene la legislazione italiana punisca molto severamente la schiavitù e la tratta di esseri umani, il reato di immigrazione clandestina, introdotto dalla L. $\mathrm{n}$. $94 / 2009$, aveva di fatto creato una situazione giuridica di assenza quasi totale di diritti per gli immigrati clandestini. Tale situazione aveva posto le premesse per uno sfruttamento di tipo schiavistico, venuto alla ribalta delle cronache in seguito a disordini verificatisi in Rosarno nel gennaio 2010. Nell'attività legale si era preso atto della impossibilità di effettuare una efficace difesa dei migranti clandestini vittime di tale situazione indegna di uno Stato di diritto. 


\section{NIEWOLNICTWO I HANDEL LUDŹMI: NOWE KATEGORIE DLA STAROŻYTNYCH PLAG}

Niewolnictwo było w prawie rzymskim statusem osobistym opartym o regulacje różne od współczesnych: głównym punktem regulacji była dominica potestas nad niewolnikami, czyli prawo właściciela co do tej szczególnej res, którą był niewolnik.

Począwszy od chrześcijańskiej koncepcji równości ludzi przed Bogiem i poprzez długą drogę prowadzącą do Uniwersalnej Deklaracji Praw Człowieka z 1948 r. doszliśmy do zakazu niewolnictwa i do stwierdzenia, że każdy człowiek jest podmiotem prawa. Jednakże, jak to twierdzi wielu wiarygodnych autorów, mamy do czynienia obecnie z nowymi formami niewolnictwa, które bazują nie na własności, ale na przetrzymywaniu ludzi, którzy stają się w ten sposób "ludzkim towarem jednorazowego użytku".

Pomimo że prawo włoskie surowo karze niewolnictwo i handel ludźmi, przestępstwo bezdomnej imigracji, wprowadzone przez Ustawę nr 94/2009, stworzyło poniekąd sytuację prawie całkowitego braku praw dla bezdomnych imigrantów. Taka sytuacja stworzyła podstawy pod wykorzystywanie w stylu niewolnictwa, o którym mówiło się wyraźnie w mediach po rozruchach w Rosarno w styczniu 2010. Podczas czynności prawnych zdano sobie sprawę z niemożliwości skutecznej obrony bezdomnych imigrantów, ofiar tej sytuacji niegodnej Państwa prawa. 


\section{SLAVERY AND TRAFFICKING IN HUMAN BEINGS: NEW CATEGORIES FOR ANCIENT PLAGUES}

Slavery in Roman law was a personal status based on provisions different from those which exist today: the main point of regulation was dominica potestas over the slaves, or the right of ownership of a particular res - which was a slave.

Starting with the Christian concept of equality of men before God, and along the long and arduous road leading to the 1948 Universal Declaration of Human Rights, we arrived at the prohibition of slavery and to the conclusion that every man is a subject of law. However, as is claimed by a multitude of reliable authors, we are now dealing with new forms of slavery, which are based not on property, but on detaining people who are in the way, "human disposable merchandise".

Although Italian law severely penalizes slavery and human trafficking, the offence of being a homeless immigrant, introduced by Law No 94/2009, created an almost total absence of rights for homeless immigrants - as in the style of slavery - of which the media spoke out clearly and positively after the Rosarno riots in January 2010. While taking legal action on their behalf, one started to be aware of the impossibility of an effective defense for homeless immigrants, who are undeserving victims of the state of law.

Sharp criticism of the legal situation effecting homeless immigrants in Italy gave rise in April 2011, to the withdrawal of the Italian regulation concerning the offence of homelessness by the Court of Justice of the European Union.

Key words: homeless immigrants, rights, law, slavery, victim 